\title{
TU/e emonown

\section{Measurements of diverging ion motion in an inertial electrostatic confinement device using Doppler spectroscopy}

\section{Citation for published version (APA):}

Hermens, J., Jaspers, R., \& Khachan, J. (2019). Measurements of diverging ion motion in an inertial electrostatic confinement device using Doppler spectroscopy. Physics of Plasmas, 26(10), [102703].

https://doi.org/10.1063/1.5119285

DOI:

$10.1063 / 1.5119285$

Document status and date:

Published: 01/10/2019

\section{Document Version:}

Publisher's PDF, also known as Version of Record (includes final page, issue and volume numbers)

\section{Please check the document version of this publication:}

- A submitted manuscript is the version of the article upon submission and before peer-review. There can be important differences between the submitted version and the official published version of record. People interested in the research are advised to contact the author for the final version of the publication, or visit the $\mathrm{DOI}$ to the publisher's website.

- The final author version and the galley proof are versions of the publication after peer review.

- The final published version features the final layout of the paper including the volume, issue and page numbers.

Link to publication

\section{General rights}

Copyright and moral rights for the publications made accessible in the public portal are retained by the authors and/or other copyright owners and it is a condition of accessing publications that users recognise and abide by the legal requirements associated with these rights.

- Users may download and print one copy of any publication from the public portal for the purpose of private study or research.

- You may not further distribute the material or use it for any profit-making activity or commercial gain

- You may freely distribute the URL identifying the publication in the public portal.

If the publication is distributed under the terms of Article 25fa of the Dutch Copyright Act, indicated by the "Taverne" license above, please follow below link for the End User Agreement:

www.tue.nl/taverne

Take down policy

If you believe that this document breaches copyright please contact us at:

openaccess@tue.nl

providing details and we will investigate your claim. 


\section{Measurements of diverging ion motion in an inertial electrostatic confinement device using Doppler spectroscopy}

Cite as: Phys. Plasmas 26, 102703 (2019); https://doi.org/10.1063/1.5119285

Submitted: 10 July 2019 . Accepted: 14 September 2019. Published Online: 04 October 2019

J. Hermens (D), R. Jaspers (D), and J. Khachan

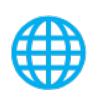

View Online

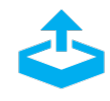

Export Citation

\section{ARTICLES YOU MAY BE INTERESTED IN}

\section{LV-DIB-s4PT: A new tool for astrochemistry}

Review of Scientific Instruments 90, 103101 (2019); https://doi.org/10.1063/1.5116925

Study of drop impact on thin fibers helps with net design for water collection

Scilight 2019, 401102 (2019); https://doi.org/10.1063/10.0000127

Collision frequency and mean free path for plasmas described by kappa distributions

AIP Advances 9, 105307 (2019); https://doi.org/10.1063/1.5125714

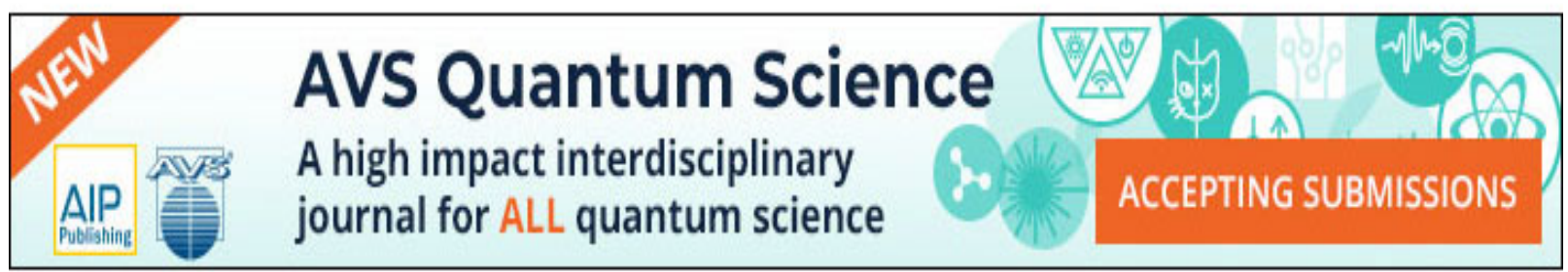




\title{
Measurements of diverging ion motion in an inertial electrostatic confinement device using Doppler spectroscopy
}

Cite as: Phys. Plasmas 26, 102703 (2019); doi: 10.1063/1.5119285

Submitted: 10 July 2019 - Accepted: 14 September 2019 •

Published Online: 4 October 2019

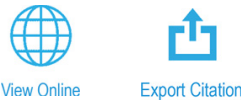

J. Hermens, ' (D R. Jaspers, ' iD and J. Khachan ${ }^{2}$

\author{
AFFILIATIONS \\ 'Departement of Applied Physics, Eindhoven University of Technology, Eindhoven 5600 MB, The Netherlands \\ ${ }^{2}$ School of Physics, University of Sydney, Sydney NSW 2006, Australia
}

\begin{abstract}
With the aim of determining the ion direction, Doppler spectroscopy is carried out in a Farnsworth fusor with a spherical cathode grid during star mode operation. The Doppler shift of Balmer- $\alpha$ radiation from excited deuterium atoms $\left(D^{*}\right)$ is analyzed to find the kinetic energy spectrum. Diverging $D^{*}$ is measured at a cathode voltage of $-20 \mathrm{kV}$, with currents between $0.5 \mathrm{~mA}$ and $3.4 \mathrm{~mA}$, and in the pressure regime of $1.6 \mathrm{~Pa}-2.6 \mathrm{~Pa}$. $\mathrm{D}^{*}$ is produced during a charge exchange reaction of fast deuterium ions with the background gas. The measured spectra can only be formed via diverging ions due to momentum conservation during this charge exchange reaction. Dominant blue shifts coming from measurement locations inside microchannels moving toward the observer and red shifted spectra coming from microchannels moving away from the observer prove this diverging ion motion. A kinetic energy distribution of $D^{*}$ over different longitudinal positions inside the microchannel is measured at a pressure of $(1.7 \pm 0.1) \mathrm{Pa}$. The results indicate an increase in $D^{*}$ velocity from the center of the cathode toward the cathode edge. This can be explained by a virtual anode, which accelerates ions created in the center of the cathode outward. These results disagree with the converging ion model but could be explained by the hollow cathode model.
\end{abstract}

Published under license by AIP Publishing. https://doi.org/10.1063/1.5119285

\section{INTRODUCTION}

Inertial electrostatic confinement (IEC) is a technique to trap charged nuclei in a spherically or cylindrically symmetric electrostatic potential well. Farnsworth used the concept of IEC to develop a gridded IEC device named the Farnsworth fusor that produced fusion of light nuclei by accelerating ions in an electrostatic potential well. ${ }^{1}$ Losses due to bremsstrahlung and collisional scattering prevent the device from producing net energy. ${ }^{2,3}$ The Farnsworth fusor has the potential of being a portable neutron source. ${ }^{4}$ Research efforts into fusion devices have shifted toward magnetic confinement fusion because it demonstrates much higher potential to produce a net-energy gain than IEC devices. Nonetheless, the fundamental physics underlying IEC fusion devices has been debated over the years; Gu et al. investigated a virtual anode in the center of a gridded cathode using proton measurements, ${ }^{5}$ Shrier et al. found diverging ion motion from the center of biconical and two ring cathodes, which contravenes the conventional view presented in the literature of a spherically converging ion focus model. However, previous publications showing diverging ion motion use grids with cylindrical symmetry. In this paper, we measure the direction of ion flow for a spherically symmetric grid, of the type used in the
Farnsworth fusor. ${ }^{6,7}$ Experiments on the direction of ion flow are presented using Doppler spectroscopy on the Balmer- $\alpha$ of deuterium to analyze if converging or diverging ion motion occurs inside a Farnsworth fusor with a spherical cathode grid.

\section{CONVERGING VS DIVERGING ION MOTION}

In gridded IEC devices, a discharge is formed between the grounded vacuum vessel and the highly negatively charged gridded cathode in the center. The vacuum potential creates an electric field that accelerates ions created between the vacuum vessel and cathode toward the center and electrons toward the vacuum vessel. The volume outside the cathode is much larger than the volume inside the cathode, and hence, if all ions created outside the cathode pass through the middle, a large positive space charge accumulates in the center. However, increased velocity of ions in the center counters the space charge accumulation. ${ }^{5}$ Nevertheless, the net effect results in a virtual anode. In Fig. 1, the electrostatic potential is illustrated and the ion motion is depicted in red. In this case, the virtual anode at the cathode center is caused by the charge accumulation of the converging ions. 


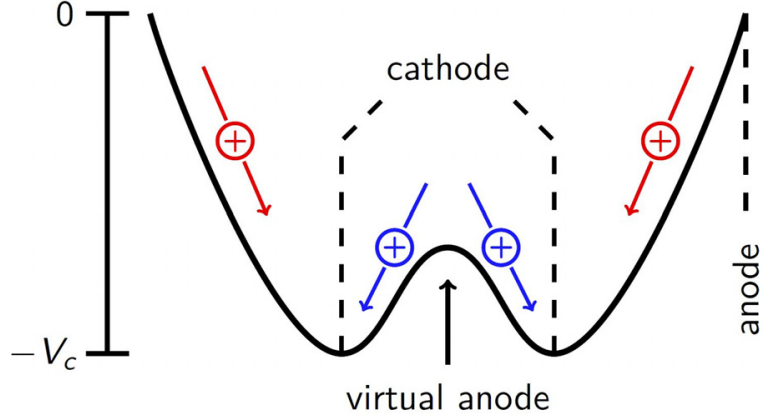

FIG. 1. The electrostatic potential inside a Farnsworth fusor. Ions created between the anode and the cathode move inward, shown in red, and ions created inside the cathode move outward, shown in blue.

This converging ion model explaining the virtual anode dependence is commonly accepted in the IEC literature. ${ }^{1,5,8}$

However, ions have been measured to predominantly leave the cathode in certain regimes, instead of entering or passing through the cathode. ${ }^{6,7,9}$ An idea is proposed to explain this behavior, which is called the hollow cathode model..$^{10}$ In the hollow cathode model, one expects to find most ionization reactions inside the cathode because of the hollow cathode effect; the pendulum motion of the electrons inside the hollow cathode causes additional ionization reactions, ${ }^{11}$ after which electrons will leave the cathode faster than the ions because of the difference in mobility. What is left is a surplus of positive space charge called a virtual anode. This virtual anode can accelerate ions outward after which, in certain pressure regimes, the ions undergo charge exchange with the background gas and continue on a straight line trajectory out of the grid as excited neutral atoms $\left(D^{*}\right)$. This explains ions predominantly leaving the cathode. The expected ion motion in the hollow cathode model is illustrated by the blue arrows in Fig. 1.

In both ion motion models, collisions of ions and electrons with the background gas can result in ionization and excitation reactions. In star mode, the microchannels with most excitation reactions are visible as shown by the light pink crossed lines in Fig. 2. The microchannels are regions of the highest electron current emission from within the grid and, therefore, produce the highest rate of ionization and

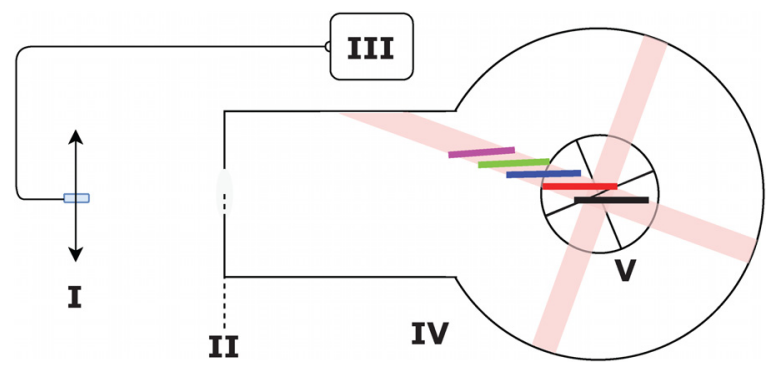

FIG. 2. Experimental setup consisting of I: optical fiber, II: positive lens, III: spectrometer, IV: vacuum chamber, and V: high voltage cathode. Microchannels are depicted in light pink. The different colored lines that pass through the microchannels correspond to the line of sights during different measurements plotted in Figs. 3 and 4. excitation of the background gas. Consequently, the highest density of energetic ions is also found in these microchannels.

\section{EXPERIMENTAL APPARATUS}

The Farnsworth fusor used during the experiments was composed of a cylindrical grounded vacuum chamber. The chamber has an inner diameter of $135 \mathrm{~mm}$ and a height of $290 \mathrm{~mm}$. A spherical stainless steel three ring grid with a diameter of $(5.0 \pm 0.2) \mathrm{cm}$ was attached to a stainless steel stalk positioned in the middle of the chamber. The stalk was insulated from the plasma with a tube of alumina ceramic. A stainless steel cylinder with a quartz window was mounted on a port. A lens was attached to the window which projected an image of the discharge optical emission onto a plane that contained a $1 \mathrm{~mm}$ optical fiber, which could be moved to collect light from different regions of the discharge image. The spectrometer had a resolution of $0.5 \AA$.

A schematic cross section of the vacuum chamber and spherical cathode grid is shown in Fig. 2. The fusor was operated in star mode. Experiments were conducted at a negative bias on the cathode of $20 \mathrm{kV}$, with currents between $0.5 \mathrm{~mA}$ and $3.4 \mathrm{~mA}$. Star mode was observed in the pressure range of 1.6-2.6 Pa. At lower pressures, an electron gun was used to sustain the discharge, which produced incandescent light from its heated tungsten filament in addition to the much weaker intensity light from the plasma that results from lower pressures. Consequently, the spectral lines could no longer be resolved. In Fig. 2, the line of sight cross sections of the microchannels for different positions of the fiber are illustrated by color matching the colors of spectra in Fig. 4. These are used to measure the Doppler shift of the deuterium Balmer- $\alpha$ line at $656.29 \mathrm{~nm}$ and, therefore, determine the ion energies along the microchannel. Balmer- $\alpha$ emission coming from outside the microchannel, but within the line of sight, can also contribute to the measured spectrum. However, this emission is imaged outside the image plane and hence has a lower contribution to the spectrum. The results will be discussed in detail in the experimental results and analysis section. However, first preliminary measurements and their conclusions are stated, which are necessary as a form of calibration.

\section{A. Calibration}

The spatial resolution of the translated optical fiber was determined by shining a light source at the grid within the chamber such that its image was formed at the optical fiber plane. The spatial resolution of the line of sight measurements was found to be $3 \mathrm{~mm}$. Translational measurements were performed perpendicular to the microchannel to validate that most Balmer- $\alpha$ emission occurred along the microchannels. Spectra were subsequently recorded by rotating the cathode through a series of angles with respect to the line of sight of the spectrometer. This shows that the Doppler shift increased with the decreasing angle, showing that any scattered light within the chamber had an insignificant effect on the behavior of the spectra. Moreover, spectra were recorded at pressures between $(0.27 \pm 0.02) \mathrm{Pa}$ and $(2.6 \pm 0.1) \mathrm{Pa}$ to determine the influence of pressure. It was found that below $(1.6 \pm 0.1) \mathrm{Pa}$, the intensity of light emission from $D^{*}$ was too low to be recorded by the spectrometer. The reduction in pressure reduces the rate of charge exchange, which resulted in optical emission that was below the threshold of the spectrometer. However, this does not imply that there was a change in the motion of ions. In this paper, 
we report on the pressure regime that produced sufficient light intensity for emission to be recorded.

\section{EXPERIMENTAL RESULTS AND ANALYSIS}

Direct observation of deuterium ions is not possible using Doppler spectroscopy. For this analysis, we depend on charge exchange reactions of fast ions with background gas, resulting in fast excited neutral atoms and molecules. Momentum is conserved during this charge exchange reaction, and hence, the direction of the fast neutral is unchanged compared to that of the fast ion. ${ }^{5}$ Three different charge exchange reactions can lead to an excited neutral as shown in the following equations:

$$
\begin{gathered}
D^{+}+D_{2} \rightarrow D^{*}+D_{2}^{+}, \\
D_{2}^{+}+D_{2} \rightarrow D^{*}+D+D_{2}^{+}, \\
D_{3}^{+}+D_{2} \rightarrow D^{*}+D_{2}+D_{2}^{+} .
\end{gathered}
$$

As deuterium is chemically equivalent to hydrogen, it is assumed that charge exchange reactions and cross sections for hydrogen and deuterium are similar. In an IEC device similar to the one used in this experiment, it was found that mostly $\mathrm{H}_{2}^{+}$and $\mathrm{H}_{3}^{+}$are present during operation. ${ }^{13,14}$ Moreover, cross sections of charge exchange reactions leading to excited neutrals increase for increasing kinetic energy. ${ }^{15}$

The recorded Doppler spectra are broadened due to multiple effects. First, ions are created at all positions within the discharge and, consequently, will accelerate through a range of potential differences, which results in an ion velocity distribution. Second, these ions do not all exchange charge at the same position, and hence, the end position of ion acceleration before charge exchange occurs is spread. Third, excited neutrals do not decay instantly, which broadens the measured spectra at any particular location. Finally, the three possible source ions have different masses, which at similar energies cause three distinguishable Doppler shifted spectral lines after charge exchange. The combination of these broadening mechanisms results in broadened peaks for each of the reactions shown in Eqs. (1)-(3). The convolution of each of these spectra results in the broadened Doppler shifted wings on either side of the unshifted Balmer- $\alpha$ line.

Presented in Fig. 3 is a measured spectrum at a cathode voltage of $-20 \mathrm{kV}$, a discharge current of $3.4 \mathrm{~mA}$, and a pressure of $(1.7 \pm 0.1) \mathrm{Pa}$ for a specific measurement location along the microchannel depicted in the figure. The line of sight has an angle of $(7.5 \pm 5)^{\circ}$ with the microchannel. Two peaks are observed, and the central one has no Doppler shift and originates from slow $D^{*}$ originating from electron excitations. The larger blue shifted shoulder originates from charge exchange reactions of fast ions. The dominant blue shift indicates that neutrals are predominantly traveling toward the observer. Hence, the direction of ion flow is away from the center, which we label as diverging ions. This predominantly blue shifted spectrum has been reproduced at pressures between $(1.6 \pm 0.1) \mathrm{Pa}$ and $(2.6 \pm 0.1) \mathrm{Pa}$. To distinguish the microchannels moving toward and moving away from the observer, the angle cannot be $0^{\circ}$. Furthermore, if the angle between the microchannel and the observer approaches $90^{\circ}$, only thermal Doppler shifts remain. Hence, the predominantly blue shifted spectrum was found at angles between $(2.5 \pm 5)^{\circ}$ and $(72.5 \pm 5)^{\circ}$. Additionally, a predominately red shifted spectrum was measured at an angle of $(162.5 \pm 5)^{\circ}$. This indicates that the asymmetry in the vacuum chamber on the side of the

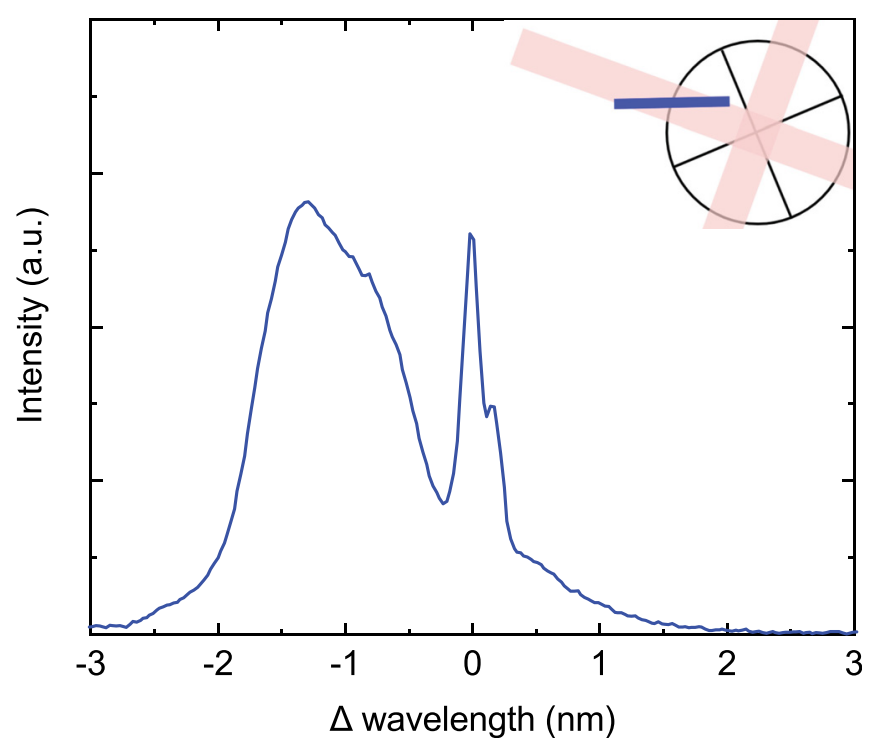

FIG. 3. Doppler shifted spectrum of $D^{*}$ with the line of sight depicted in the legend. The spectrum consists of a sharp peak in the center caused by electronically excited $D^{*}$ and a blue shifted shoulder caused by charge exchange reactions with fast ions.

observer is not the cause of the diverging ions because this ion flow is also reproduced on the other side of the cathode.

In addition, measurements of different longitudinal positions of the microchannel are plotted in Fig. 4. These measurements illustrate that a red shift is measured if the line of sight crosses the microchannel moving away from the observer and a blue shift is observed at different longitudinal positions of the microchannel moving toward the observer. Note that the red-shifted wings are greatly reduced as one

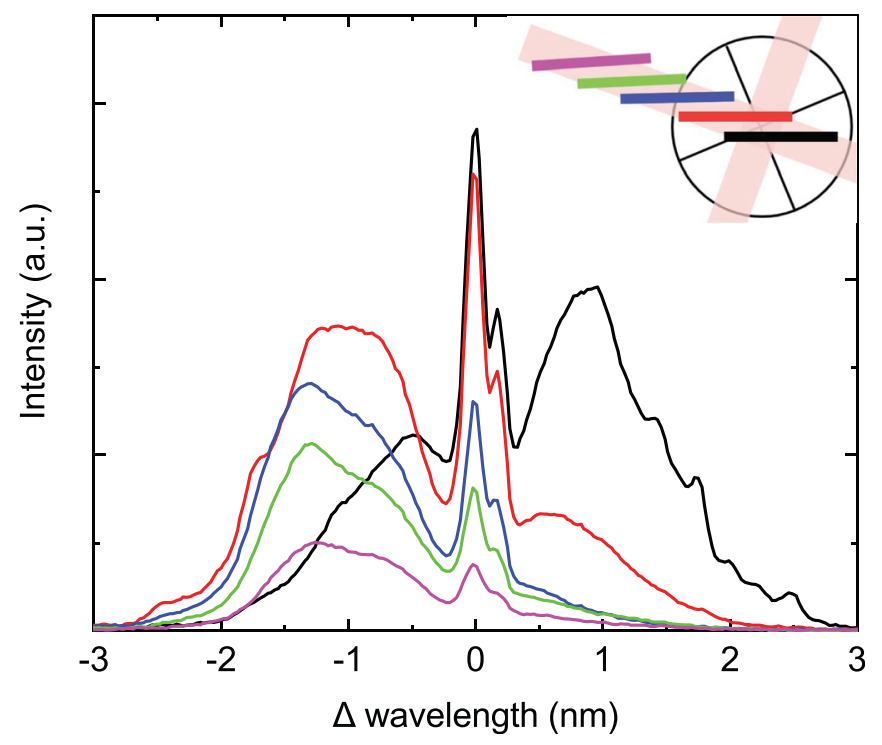

FIG. 4. Doppler shifted spectrum of $D^{*}$ at different longitudinal positions of the microchannel. 
moves away from the cathode center. This implies that the density of ions converging along a microchannel toward the center is much less than the density of diverging ions. Moreover, it counters the arguments that the diverging ions are a result of those that have converged to the center from one side of the cathode and undergone charge exchange to emerge on the other side. If this were the case with a spherically symmetric cathode, the converging ions should also be in the recorded spectra and register as a red Doppler shift but are clearly absent.

To analyze the energy of the neutrals at the measurement location, the peak intensity of the shoulder is assumed to represent the relative energy of the neutrals at that particular location since it represents the peak value of the velocity distribution. In this way, a relative comparison between the velocity distributions at different longitudinal positions of the microchannel can be made. Doppler shifted wavelengths can be converted to kinetic energy combining the kinetic energy equation $\mathscr{E}_{\kappa}=\frac{1}{2} m v^{2}$ and the non relativistic approximation for Doppler shifts $\Delta \lambda \approx \frac{v \lambda_{0}}{c} \cos \phi$, resulting in the following equation:

$$
\mathscr{E}_{\kappa}=\frac{m c^{2}(\Delta \lambda)^{2}}{2 \lambda_{0}^{2} \cos ^{2} \phi},
$$

with $\mathscr{E}_{K}$ and $\mathrm{m}$ being the kinetic energy and mass of the observed neutral, $\mathrm{c}$ the speed of light, $\Delta \lambda$ the Doppler shift, $\lambda_{0}$ the Balmer $\alpha$ wavelength of deuterium, and $\phi$ the angle between the line of sight and the microchannel. Not all measurements corresponding to longitudinal translations are plotted in Fig. 4. Moreover, the energies corresponding to the wavelengths with peak intensity values of the shoulders are plotted in Fig. 5 for all translational positions of the fiber relative to the center of the cathode. Hence, these energies correspond to the fast excited neutrals with the highest density at that measurement location. One observes low energies around the cathode center

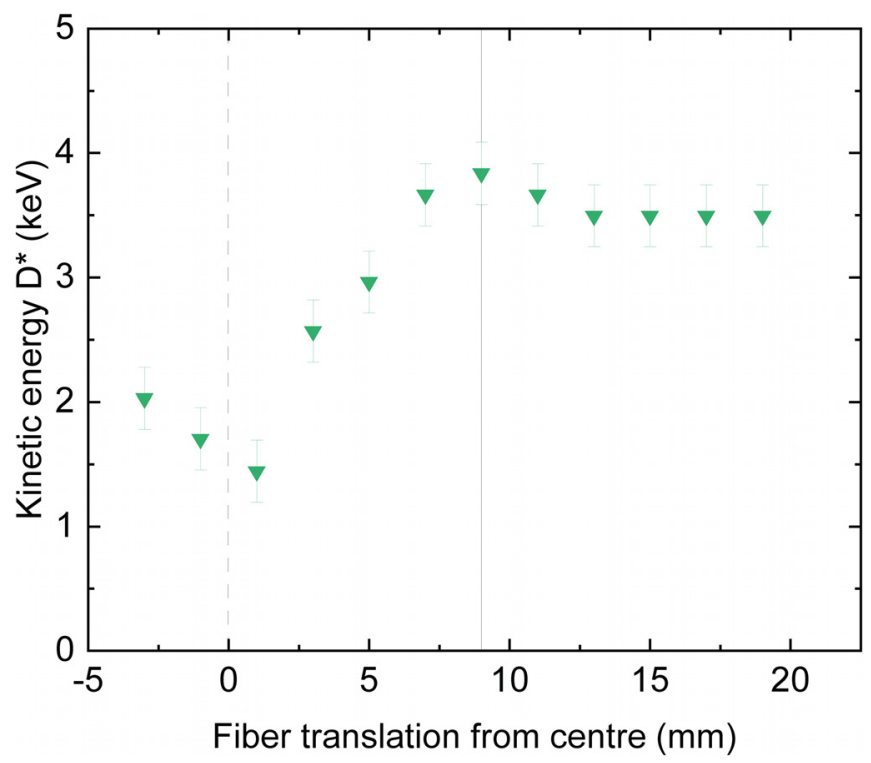

FIG. 5. The energies corresponding to the peak intensity Doppler shifts are plotted for different longitudinal positions of the star mode beam. The dashed line marks the cathode center, and the solid line marks the cathode edge. and an increase in kinetic energy moving toward the edge of the cathode. This acceleration outward can be explained by a virtual anode. However, one would expect a deceleration outside the cathode, which is not observed. This is explained by the charge exchange mean free path of the ions that is in the range of $4 \mathrm{~cm}$ at these operating conditions. By the time ions leave the cathode, most have undergone charge exchange. The ions that are left can decelerate and make a small contribution to the measured spectrum once they undergo charge exchange.

\section{DISCUSSION}

In an operating regime with mean free paths larger than the scale length of the vacuum chamber, ions would oscillate around the cathode, resulting in an approximately symmetric spectrum. However, in the relatively high pressure operating regime used here, the mean free path is too low for multiple ion oscillations before they exchange charge with the background gas. As expected, asymmetric spectra are measured with these relatively high pressures. The commonly accepted converging ion model predicts asymmetric spectra that indicate ions moving toward the center. The contrary is measured; ions predominately leave the cathode, and a reversal in the Doppler shift is observed once one crosses the cathode center. This cannot be explained via converging ions created outside the cathode. However, ions produced in the center of the cathode and accelerated outward by the virtual anode could be a valid explanation for the measurements. In the measured regime, experiments could instead be designed to increase the height of the virtual anode, to increase ion velocity, and maximize fusion. This is left for future research.

The diverging ions have only been measured in a specific pressure range. At lower pressures often used in the literature, ${ }^{1}$ collisional mean free paths increase, which influences most mechanisms involved. For example, the height and shape of the virtual anode could change, and ions would be able to oscillate in and out of the cathode before undergoing exchanging charge. Further measurements should be done outside the presented parameter scope to research ion motion in different operating regimes.

In addition, experimental parameters such as cathode geometry and material could also be of influence. Since the plasma density within the cathode does rely to some degree on secondary electron emission, the height of the virtual anode may change between different metals, which leads to a different ion energy distribution. However, ambipolar diffusion that leads to the virtual anode may have a greater dependence on the geometry of the cathode, and so it is not clear whether using different materials will have a significant effect on the energy of the diverging neutrals. Such work will need to be the topic of another study. Furthermore, with a spherical cathode grid, the amount of rings used could be of influence. However, previous experiments show similar virtual anode shapes for spherical cathodes with more cathode rings. 9,16

\section{CONCLUSION}

Diverging ions have been measured in a Farnsworth fusor with a spherical cathode grid using Doppler spectroscopy. Diverging ions were observed in star mode at pressures between $(1.6 \pm 0.1) \mathrm{Pa}$ and $(2.6 \pm 0.1) \mathrm{Pa}$ operating at $-20 \mathrm{kV}$. Measurements were carried out at different longitudinal positions of the microchannel in which a velocity evolution of the fast neutrals inside the microchannel was found. 
The increase in velocity moving away from the center of the cathode can be explained by a virtual anode accelerating ions outward. This does not agree with the commonly accepted converging ion model but could be explained by the hollow cathode model.

\section{ACKNOWLEDGMENTS}

The authors are grateful to the helpful discussions with Nico Ranson, Richard Bowden-Reid (University of Sydney), and Maximilian Messmer (University of Technology Eindhoven). This work was carried out within the framework of the EUROfusion Consortium and received funding through FuseNet from the Euratom research and training program under Grant Agreement No. 633053. The views and opinions expressed herein do not necessarily reflect those of the European Commission. In addition, funding was provided by TU/e Fund International Experience.

\section{REFERENCES}

${ }^{1}$ G. H. Miley and S. K. Murali, Inertial Electrostatic Confinement (IEC) Fusion (Springer, 2014).

${ }^{2}$ T. H. Rider, "A general critique of inertial-electrostatic confinement fusion systems,” Phys. Plasmas 2(6), 1853-1872 (1995).

${ }^{3}$ T. H. Rider, "Fundamental limitations on plasma fusion systems not in thermodynamic equilibrium," Phys. Plasmas 4(4), 1039-1046 (1997).

4J. Sved, "Commercial IEC portable neutron source," Trans. Am. Nucl. Soc. 77, 504-505 (1997), available at https://inis.iaea.org/search/search.aspx?orig_q=RN: 29021193.
${ }^{5}$ Y. Gu and G. H. Miley, "Experimental study of potential structure in a spherical IEC fusion device," IEEE Trans. Plasma Sci. 28(1), 331-346 (2000).

${ }^{6}$ O. Shrier, J. Khachan, S. Bosi, M. Fitzgerald, and N. Evans, "Diverging ion motion in an inertial electrostatic confinement discharge," Phys. Plasmas 13(1), 012703 (2006).

${ }^{7}$ J. Khachan and A. Samarian, "Dust diagnostics on an inertial electrostatic confinement discharge,” Phys. Lett. A 363(4), 297-301 (2007).

${ }^{8}$ T. Motoyasu, S. Namba, and K. Takiyama, "Measurements of localized potential profiles by LIF polarization spectroscopy in an inertial-electrostatic confinement discharge,” J. Korean Phys. Soc. 65(8), 1205-1208 (2014).

${ }^{9}$ J. Khachan, D. Moore, and S. Bosi, "Spatial distribution of ion energies in an inertial electrostatic confinement device," Phys. Plasmas 10(3), 596-599 (2003).

${ }^{10}$ J. Kipritidis, "The application of doppler $\mathrm{H} \alpha$ spectroscopy to the prediction of experimental fusion rates in a deuterium-filled inertial electrostatic confinement device," Ph.D. thesis (University of Sydney, 2009).

${ }^{11} \mathrm{G}$. Stockhausen and M. Kock, "Proof and analysis of the pendulum motion of beam electrons in a hollow cathode discharge," J. Phys. D: Appl. Phys. 34(11), 1683 (2001).

${ }^{12} \mathrm{~A}$. Wolf, "Measurement of ion velocities in the TU/e fusor plasma using LIF spectroscopy," M.S. thesis (Eindhoven University of Technology, 2015).

${ }^{13} \mathrm{M}$. Fitzgerald, J. Khachan, and S. Bosi, "Relative densities of hydrogen ion species in a hollow cathode glow discharge," Eur. Phys. J 39(1), 35-39 (2006), available at https://link.springer.com/article/10.1140/epjd/e2006-00070-x.

${ }^{14} \mathrm{C}$. Barbeau and J. Jolly, "Spectroscopic investigation of energetic atoms in a DC hydrogen glow discharge,” J. Phys. D: Appl. Phys. 23(9), 1168 (1990).

${ }^{15}$ I. Williams, J. Geddes, and H. Gilbody, "Balmer $\alpha$ emission in collisions of $\mathrm{H}$, $\mathrm{H}^{+}, \mathrm{H}_{2}^{+}$and $\mathrm{H}_{3}^{+}$with $\mathrm{H}_{2}$," J. Phys. B 15(9), 1377 (1982).

${ }^{16}$ T. A. Thorson, R. D. Durst, R. J. Fonck, and L. P. Wainwright, "Convergence, electrostatic potential, and density measurements in a spherically convergent ion focus," Phys. Plasmas 4(1), 4-15 (1997). 